\title{
1 Second Generation of Pollutant Emission Models for SUMO
}

Daniel Krajzewicz, Michael Behrisch, Peter Wagner;

German Aerospace Center, Rutherfordstraße 2, 12489 Berlin, Germany

\{Daniel.Krajzewicz, Michael.Behrisch, Peter.Wagner\}@DLR.de

Stefan Hausberger;

3130 Institut für Verbrennungskraftmaschinen und Thermodynamik, Inffeldgasse 19/I (Emissionsforschung), $8010 \mathrm{Graz,}$ Austria

hausberger@ivt.tugraz.at

Mario Krumnow;

Technische Universität Dresden, Institut für Verkehrstelematik, 01062 Dresden, Germany

Mario.Krumnow@tu-dresden.de

\subsection{Abstract}

Traffic puts a high burden on the environment in means of emitted pollutants and consumed fuel. Different attempts exist for reducing these impacts, ranging from traffic management actions to in-vehicle ITS solutions. When equipped with a model for vehicular pollutant emissions, microscopic traffic simulations are assumed to be helpful in predicting the performance of such approaches. We report about the implementation of a second generation of pollutant emission models.

Keywords: emissions, modelling, environment, traffic management.

\subsection{Introduction}

Air pollution is a well-known problem that ranges from local air quality issues up to global effects the humanity is confronted to. Following the International Transport Forum ([1]), "[the] Transport-sector $\mathrm{CO}_{2}$ emissions represent 23\% (globally) and 30\% (OECD) of overall $\mathrm{CO}_{2}$ emissions from Fossil fuel combustion. The sector accounts for approximately $15 \%$ of overall greenhouse gas emissions." Different actors are involved in reducing road traffic's environmental impact and resource consumption, often forced to do so by law. In Europe, automobile manufacturers shall reduce their fleet emissions [2]. Cities try to keep the amounts of pollutant concentrations below the thresholds formulated in according regulations, such as [3]. Finally, pollutant generation is closely coupled with the consumption of fuel. As fuel price has increased in the past years the reduction of pollutants is also in the focus of end users - individuals as well as (e.g. logistics) companies. 
The development of technical solutions for critical systems usually includes a step where the solution is modelled and simulated. This step allows to validate the assumptions about the solution's functionality and to a-priori benchmark or proof its performance. Traffic simulations are an established tool used by both, consultants and researchers. Conventionally, microscopic models are applied to areas that cover few intersections, while macroscopic models are used to replicate areas at city level. Academic approaches, such as the traffic simulation SUMO $[4,5]$ that is discussed herein, attempt to simulate large, city-wide areas using microscopic models.

In any case, for evaluating a solution that was designed to reduce road traffic's impact on the air quality, the involved traffic simulation must be capable to compute the amount the emitted pollutants to reduce with a predictable error. A large variety of emission models is described in the scientific literature. They differ in the required input parameters, the covered pollutants, the coverage of the real-world vehicle population (regarding their emission behavior), and the aggregation of the results in time and area. Therefore, it is necessary to formulate the requirements before choosing a model to implement.

In the following, the recent work on vehicular emissions modelling in SUMO will be presented. This work has been performed within the projects "COLOMBO" [6, 7] and "AMITRAN" [8, 9]. The models implemented within these projects replace SUMO's initial emissions model that was developed within the project "iTETRIS" [10, 11]. All three project are or respectively were co-funded by the European Commission.

The remainder is structured as following. A discussion of SUMO's requirements to an emission model is given, first, followed by an overview about emissions modelling and available emission models. A description of the implemented emission models into SUMO is given afterwards. Then, using and extending the emission models embedded in SUMO is described. Some use cases are presented afterwards. The report ends with a summary.

\subsection{SUMO's Requirements to an Emissions Model}

Briefly said, the model to choose should be capable to be used as a further measurement within the applications the traffic simulation is usually used for. As SUMO's goal is to simulate real-world traffic in large areas, the model should cover the population of vehicles found on roads nowadays. This counts for passenger vehicles as well as for heavy duty vehicles, busses, motorcycles, etc. One should also take into regard that the deployment of currently developed ITS applications will be realized in the future. Therefore, the model should be capable to represent future compositions of vehicle population. Some types of investigations require a distinction of regulative emission classes, e.g. the EURO-norm. Such a classification also helps in representing the population of vehicles over time, as most statistics on past and current vehicle fleets are represented this way.

A second top-level requirement is that the emission model should match the resolution of the traffic simulation. It should be sensible to all vehicle (or traffic) state attributes that are available in the simulation. In case of a microscopic simulation, a vehicle's acceleration, speed, and the slope of the road beneath the vehicle are the major attributes to consider. On the contrary, the model is wanted to use only parameters that are offered by the traffic simulation model. Such a close connection to the traffic model implies the possibility to compute emission values for each simulated time step, usually having a length of $1 \mathrm{~s}$. To achieve this, the emissions model must allow to compute emissions at such a time scale.

Not all available models cover all pollutants emitted by road traffic. Therefore the pollutants assumed to be needed should be defined. Within the iTETRIS project (see [12]), it was decided to model the emission of $\mathrm{CO}, \mathrm{CO}_{2}, \mathrm{NO}_{x}, \mathrm{PM}_{\mathrm{x}}$, and $\mathrm{HC}$, because these emissions are toxic (CO), 
cause cancer $\left(\mathrm{PM}_{\mathrm{x}}\right)$, are responsible for ground-level ozone increase and smog generation $\left(\mathrm{NO}_{x}\right.$ and $\left.\mathrm{HC}\right)$ or are greenhouse gases $\left(\mathrm{CO}_{2}\right)$. Additionally, the fuel consumption should have been modelled.

The model has to fulfil some other, non-functional requirements. It should be portable matching SUMO's overall portability. It should be fast in execution for not prohibiting its usage in large-scale scenarios. And it should be directly embedded into the simulation to avoid additional interaction (e.g. socket-based) or file exchange.

SUMO's viral GPL license requires the implementation of the model to be made available under the same license. And, of course, the model should be easily usable. Summarizing, the following requirements are put on the model:

- Cover the complete vehicle population (in means of emission classes);

- Offer a classification of classes into EURO-norms;

- Compute certain pollutants $\left(\mathrm{CO}, \mathrm{CO}_{2}, \mathrm{NO}_{x}, \mathrm{PM}_{\mathrm{x}}, \mathrm{HC}\right.$, and fuel consumption were chosen);

- (Be) sensible to microscopic parameters available in the simulation;

- Require only information that is available in the simulation;

- $\quad(\mathrm{Be})$ able to compute emissions in simulated time steps;

- $(\mathrm{Be})$ easy to parameterize:

- $(\mathrm{Be})$ portable, fast in execution, and directly embedded into the simulation;

- (Be) licensed under a GPL-compatible license.

\subsection{Emission Models Overview}

Most of nowadays vehicles burn petroleum-derived fuel for propulsion. When regarding small time scales ${ }^{1}$, fuel consumption depends on the vehicle's engine characteristics as well as on the current load on the engine. The load is dictated by the force a vehicle needs to overcome as well as by the chosen gear (see [13] for a good explanation). The majority of the fuel burns to $\mathrm{CO}_{2}$ and water, but other, often poisonous gases are generated as well. Catalytic converters convert a major portion of some of these pollutants into non-poisonous gases. The performance of the catalytic converter mainly depends on the catalyst's temperature as well as on the engine's current operating point. Different other influences exist, such as drive train losses, the road's slope, or the air-fuel ratio at combustion to name a few. Additionally, longterm effects of certain driving styles may change a vehicle's emission behavior.

In summary, every vehicle has an individual emission behavior. But when investigating road traffic, many vehicles of different types have to be regarded. It is thereby necessary to find a tradeoff between the amount of vehicle emission classes a model covers and the details in modelling each single vehicle or emission class. The literature accordingly distinguishes the following major emission model classes:

- "inventory" emission models that include data for the major portion of the vehicle emission classes; their input usually includes a vehicle population composition and the amount of driven distance, optionally also the average speed or an abstract traffic state. Such models usually cover a large set of different pollutants.

- "instantaneous" (or "modal") emission models that simulate a single vehicle's emission, where [14] proposes a further distinction into emission maps, regressionbased models, and load-based models. Trying to model the emissions for a single

\footnotetext{
${ }^{1}$ Most instantaneous consumption models work with steps of 1 second
} 
vehicle as exact as possible, these models usually regard a small number of vehicles only.

As one can see, the models differ in granularity and the input parameters they need as well as in the number of pollutants covered. One should note that some "instantaneous" models exist which databases were incrementally extended over the years to cover a large portion of real-world's vehicle emission classes. The model PHEM ("Passenger and Heavy Vehicles Emission Model") $[15,16]$ which derivate was included in SUMO, see section 1.5.2, is one of such models. Its sub modules and their inter-dependencies are shown in Figure 1-1.

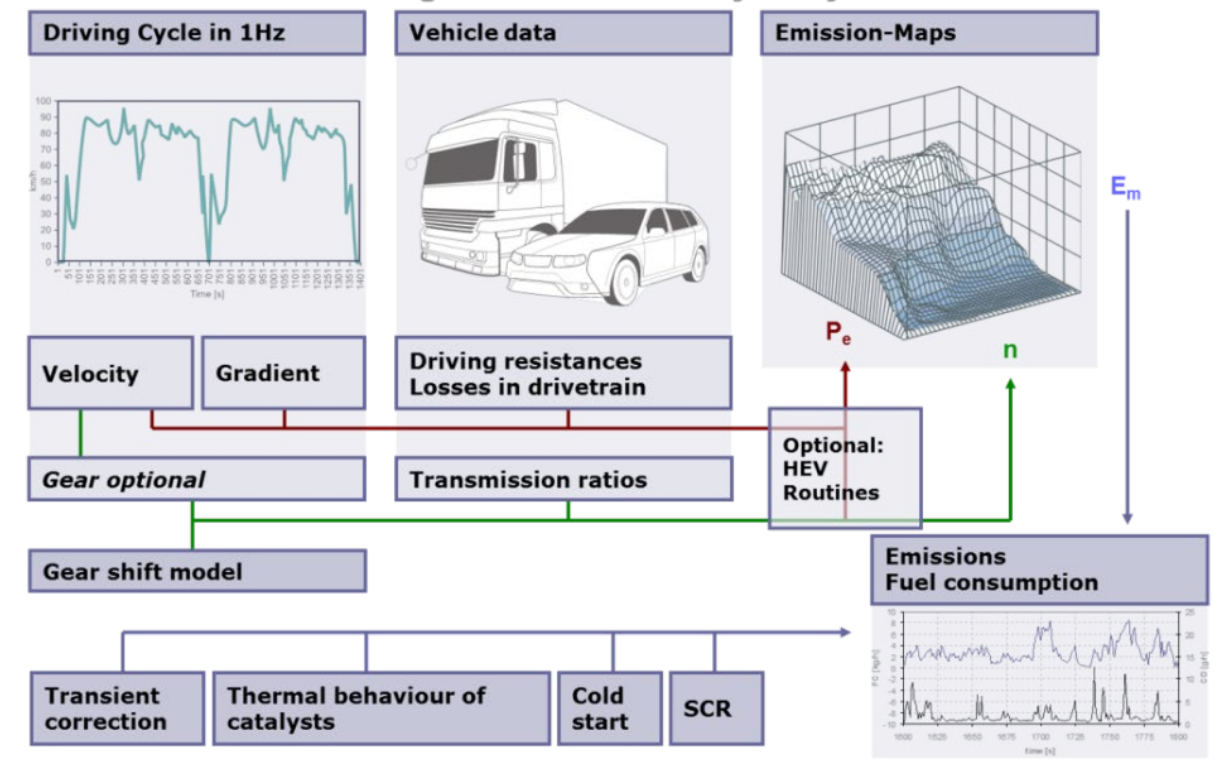

Figure 1-1: Schematic representation of the PHEM emission model [18].

Within the iTETRIS project, 15 non-commercial (freely available in means of data or a document that completely defines them) emission models have been investigated to determine candidates for being embedded into SUMO. Commercial models have not been included in this investigation. None of the 15 models fulfilled the posed requirements directly. The inventory models were found to be too coarse due to being insensitive to the vehicle's acceleration. Most microscopic models either did not include methods for computing all of the desired pollutants or the needed input parameters were not completely given or would introduce a high number of additional parameter into SUMO's vehicle type description.

\subsection{Implemented Emission Models}

As no emission model could be found that is instantaneous in means of regarding vehicle attributes used in a microscopic simulation but still covering a major part of the vehicle population, the decision to build an own model based on data from the HBEFA [17] database was taken. HBEFA, in version 2.1 at that time, is one of the investigated inventory models. This initial implementation of an emission model in SUMO will be described in the following sub-section. Two recently developed emission models will be presented afterwards: PHEMlight which is derived from PHEM and a new approach to reformulate the emissions inventory database HBEFA in its version 3.1. The models have been implemented in the projects "COLOMBO" and "AMITRAN", respectively. 


\subsubsection{Initial HBEFA v2.1 Derivation}

The model was implemented by extracting the data from HBEFA and fitting them to a continuous function that was obtained by simplifying the function of the power the vehicle engine must produce to overcome the driving resistance force. The simplified function is given as $(1-1)$ :

$$
c_{0}+c_{1} v a+c_{2} v a^{2}+c_{3} v+c_{4} v^{2}+c_{5} v^{3}
$$

The same functional form has been used for all emission types, only the parameters change per emission type and vehicle. HBEFA's lack of a dependency on acceleration was compensated by using the contained information about the dependency of the emissions on the road slope. But it should be noted that the only values up to $\pm 0.6 \mathrm{~m} / \mathrm{s}^{2}$ can be determined this way, the dependency on higher acceleration/deceleration was obtained by extrapolating given values. The used version 2.1 of HBEFA lacked data for rare vehicle classes (e.g. EuroNorm- 6 vehicles at that time). Both low as well as high velocities, the latter mainly for heavy duty vehicles, were missing for some emission classes as well. Therefore, the obtained curves did not match some basic emission properties, such as being always above zero or producing emissions at a velocity of $0 \mathrm{~m} / \mathrm{s}$. Emission classes that were recognized to be badly represented by the fitted function were removed.

The so obtained curves for the remaining vehicle classes were clustered into groups of similar behavior. The initial idea for performing this step was to reduce the number of emission classes to reduce the effort needed to set up a simulation scenario. In fact, this attempted simplification yielded in a set of badly usable emission classes. The lack of an explicitly given Euro-norm does not allow investigations of regulatory actions such as environmental zones that distinguish between vehicles of a certain emission class and the lack of a projection from the clusters back to the original classes makes setting up a realistic emission population complicated.

These issues were regarded during the implementation of the new HBEFA-based emission model described in section 1.5.3.

\subsubsection{PHEMlight}

PHEMlight is a simplified version of the emission model PHEM. PHEMlight has been designed and implemented by the Technical University of Graz within the COLOMBO project. PHEM itself provides basic emission factors for HBEFA 3 and COPERT and thus can be regarded as an de facto European reference.

PHEMlight uses characteristic emission curves which define the emission amount $[\mathrm{g} / \mathrm{h}]$ as function of the actual engine power of the vehicle. These characteristic curves were computed using PHEM with representative dynamic real word driving cycles. The amounts of emissions produced by a vehicle (as well as the amount of consumed fuel) during a simulation step is determined by computing the power needed by the vehicle as shown in the following Figure 1-2, first. Then, this value is used to look up the characteristic emission curves. 


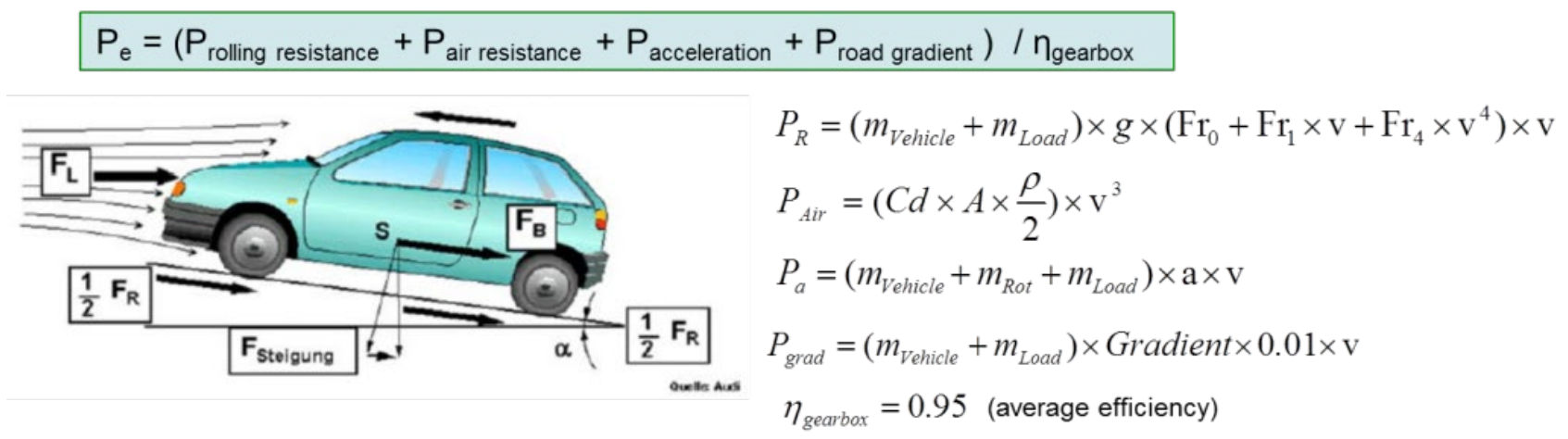

Figure 1-2: Computation of the needed propulsion power in PHEMlight [18].

PHEMlight is available as a commercial add-on. The implementation itself is included in the usual SUMO version. But the major information is stored in the characteristic curves' and vehicle attribute files. Only two emission classes are included in SUMO's open source release: an Euro-4 passenger car with a gasoline engine and a passenger car with the same emission class, but running on Diesel. The remaining emission classes have to be purchased from the Technical University of Graz.

\subsubsection{HBEFA v3.1 Derivation}

Given the lessons learned while implementing and using the initial HBEFA v2.1-based emission model and the availability of a new HBEFA version that includes new measures for modern Euro-Norm- 6 vehicles, a new attempt to build an emission model was done in the scope of the AMITRAN project.

The basic procedure is similar to the one used for the initial HBEFA derivation: values included in HBEFA are extracted for each emission class and the function (1-1) is fitted against them. Again, the slope information given in HBEFA was used to take the part of the missing dependency on acceleration. The restrictions concerning available acceleration values remain as in the initial implementation.

Fitting the values to the given function is a linear problem, since only the linear coefficients $c_{0}$ to $c_{5}$ need to be evaluated. The fitting was performed using a linear model estimation algorithm from Python's "statsmodels" package. Since a linear fit usually does not lead to a clear answer whether or not a coefficient is zero, a couple of slightly different models is tested in each case (one emission class and one vehicle class), where some of the coefficients of (1-1) are set to zero and are not estimated in the fit. By comparing these candidate functions, the best one (based on RMS and t-value) is used as the final result, i.e. a set of fitting parameters for this case at hand. This works quite well in most of the cases, the remaining challenges are that not all emissions seem well represented by (1-1).

In principle, emission curves could be fit to all emission classes included in HBEFA's version 3.1 resulting in coefficients for some hundred different emission classes in SUMO. It was but decided to use only the most common emission classes.

\subsubsection{Comparisons}

In a first step, fulfilling the requirements formulated in section 1.3 by the models is presented. It should be mentioned that all models compute the desired pollutants' emissions $\left(\mathrm{CO}, \mathrm{CO}_{2}\right.$, $\mathrm{NO}_{\mathrm{x}}, \mathrm{PM}_{\mathrm{x}}, \mathrm{HC}$, and fuel consumption). Table 1-1 shows a summary of other named requirements. 
Table 1-1: A comparison of features for the three implemented models.

\begin{tabular}{|c|c|c|c|}
\hline Requirement & HBEFA 2.1-based & HBEFA 3.1-based & PHEMlight \\
\hline No. of emission classes & $56 * 2(+1)$ & $45(+1)$ & $112(+1)$ \\
\hline coverage & $\begin{array}{l}\text { no modern (Euro 6) } \\
\text { and seldom classes }\end{array}$ & $\begin{array}{l}\text { Major passenger, heavy } \\
\text { duty, and bus classes }\end{array}$ & almost complete \\
\hline Euro-Norms & - & $x$ & $x$ \\
\hline Covers chosen polutants & $x$ & $x$ & $x$ \\
\hline Uses speed & $x$ & $x$ & $x$ \\
\hline Uses acceleration & $x$ & $x$ & $x$ \\
\hline Uses slope & - & - & $x$ \\
\hline Needs further attributes & - & - & - (are included) \\
\hline Step-size resolution & $x$ & $x$ & $x$ \\
\hline Easy parameterization & $x$ & $x$ & $x$ \\
\hline
\end{tabular}

The number of respectively covered emission classes requires an explanation. The " +1 " denotes that each model includes an emission class that does not produce emissions ("zero_emissions"). The initial model derived from HBEFA v2.1 duplicates all vehicle classes, where the second set ignores the current acceleration. These acceleration-free models were used within the investigations on emission-optimal routing (see section 1.7.2). The model does not include 56 distinct emission classes, but rather classification scheme clusters of such. Passenger vehicles can be chosen from three sets that include 3, 6, and 12 emission classes, respectively. Clusters with 7 and 14 emission types can be used for modelling heavy duty vehicles. As mentioned in section 1.5.3, the number of emission classes in the HBEFA 3.1based model could be increased when necessary.

As discussed, it is hardly possible to give a comparison of the models against real-world data as such are not available for a complete vehicular population. Currently under investigation are comparisons against a single Euro 4 passenger car emissions as well as comparisons against PHEM.

\subsection{Working with SUMO's Emission Models}

\subsubsection{User Interaction}

The implementation tries to give the user the highest grade of flexibility by allowing him to compose the vehicle fleet using the implemented emission classes. Each vehicle type can be assigned to a dedicated emission class. A vehicle type can be shared by an arbitrary number of vehicles. Emission computation is performed as soon as the user a) asks for an according output, b) asks to visualize the emissions, and/or c) asks for a vehicle's current emissions via $\mathrm{TraCl}$. All these interfaces cover all of the modelled emissions and - ignoring the visualization of emissions - are available in both, the command line and the graphical version of the simulation. The available outputs include:

- aggregation of emissions per lane with variable interval time spans

- aggregation of emissions per edge with variable interval time spans

- aggregation of emissions for each simulated vehicle

- non-aggregated (step-wise) vehicle emissions

- a vehicular trajectory file as defined in AMITRAN 
In addition, SUMO's on-line interaction interface "TraCl" allows to retrieve the emissions a single vehicle "produced" in the last simulation step, as well as emissions produced on edges or lanes. The visualization allows to color lanes and/or vehicles by the amount of pollutants emitted on them or generated by them, respectively.
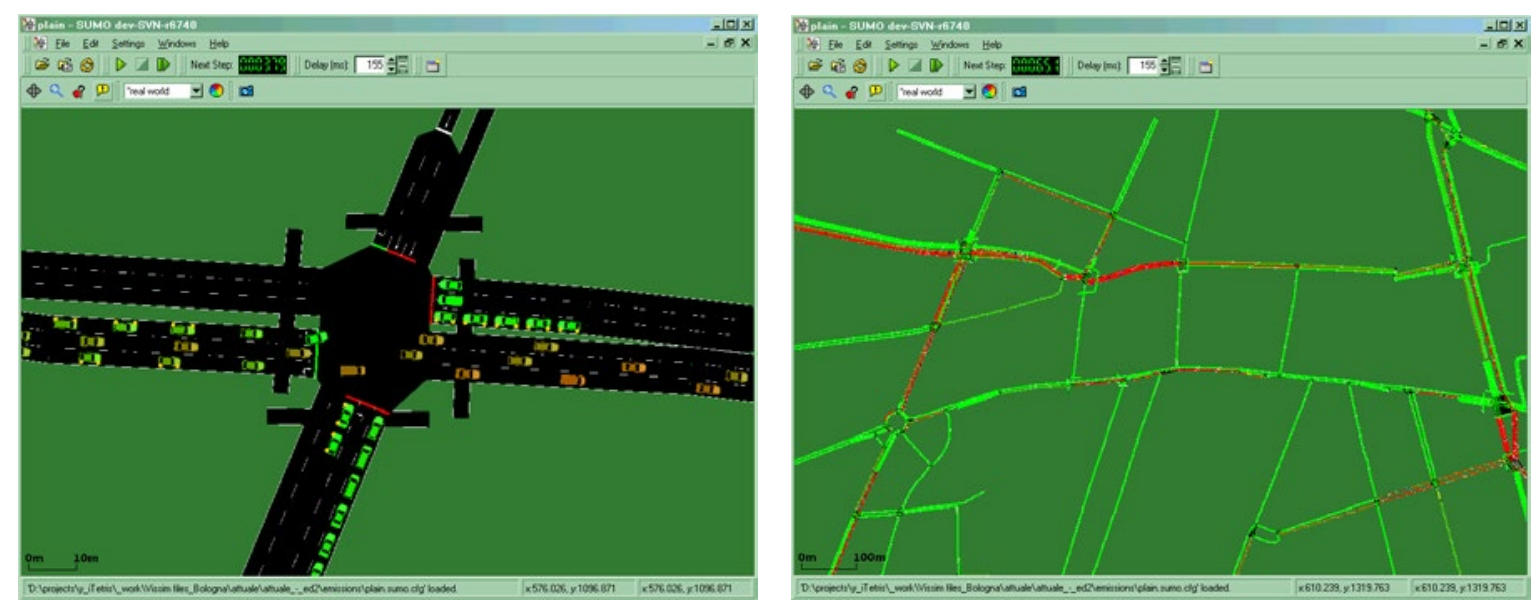

Figure 1-2: Examples of emissions visualization in SUMO; left: $\mathrm{CO}_{2}$ emitted in the last time step by individual vehicles, right: lanes colored by the amount of fuel consumed in the last step by vehicles that were on the respective lane [12].

The AMITRAN trajectory file is an intermediate data exchange file that may be converted into inputs for emission models such as VERSIT+, PHEM, and HBEFA. It is interchangeably usable among different traffic simulation ecosystems such as SUMO, VISSIM and TNO ITS Modeler. A similar approach was used to generate input files for the PHEM emission model: a converter script was set up that obtains an "fcd-output" as generated by SUMO and converts it to files that resemble the vehicle fleet, the road network, and the trajectories as read by PHEM.

SUMO's user documentation includes a description of the output functionalities and was as well extended by a chapter on emissions modelling.

\subsubsection{Router Support}

Besides enabling the traffic simulation to compute pollutant emissions, the route computation applications included in the SUMO suite were extended. The wish was to perform route computation based on the amount of emitted pollutants instead of the conventionally used travel time. To achieve this purpose, the shortest-path router was extended to read time lines of vehicular emissions as weights of edges of the road network graph. The implementations of the shortest-path algorithms were extended to use these read values as edge weights, but additionally keep track of the travel time to obtain these weights from the correct time slice of the loaded time line. This extension has already been used for different purposes, see section 1.7.2.

\subsubsection{Tools}

Several additional tools support the development and usage of emission models in SUMO context.

"emissionsDrivingCycle" takes trajectories consisting of speed, acceleration (optional), and slope (optional) for each time step of a virtually driven drive cycle for one or multiple vehicles and computes the according emissions. The obtained emission time lines can be visualized using additionally available scripts. The tool as well reads trajectories in the AMITRAN format 
mentioned above and can thus be employed to use SUMO's emission models with trajectories from other simulation tools.

"emissionsMap" computes a matrix that contains the emission amounts of modeled pollutants in dependence to a driven speed, acceleration, and slope for a named emission class. Additional visualization script allow to investigate the so obtained matrices.

\subsubsection{Embedding new Emission Models into SUMO}

The co-existence of different emission models was realized by deriving a common "interface". The interface is kept very simple. For each known pollutant, a method exists that returns its computed emission amount in $\mathrm{mg} / \mathrm{s}$ ( $\mathrm{ml} / \mathrm{s}$ for fuel). The method obtains the vehicle's emission class, its speed, acceleration, and the slope of the road it drives at. The model to use is encoded in the internal representation of the emission class which uses a 32 bit integer where the upper 16 bits are used to encode the used models while the lower 15 bits define a single emission class within this model. Bit 15 (the $16^{\text {th }}$ bit) denotes whether the regarded emission type is a heavy duty or a light (passenger) vehicle. This information is needed to compute the vehicles' noise emissions using the embedded Harmonoise noise emission model [19].

When being asked to compute the amount of a pollutant emissions, the interface determines the model to use based on the upper 16 bits, first. Then it asks the model implementation for computing the emission amount, passing all given values.

Besides giving access to the emission computation, the interface holds several further methods, mainly for computing parameters needed for file exchange between AMITRAN tools. As SUMO does not force emission models to fulfill a common view on emission classes, these methods derive information such as the fuel type, the Euro Norm, or the type of the vehicle based on the information known to the emission model implementations only.

The interface offers a clean access to the implemented models, but it should be noted that currently only models that rely on the selected parameters - emission class, speed, acceleration, and slope - can be implemented. As soon as other parameter have to be taken into account, the interface would have to be extended.

\subsection{Use Cases}

Being available for several years, the emission models have been already used in a large variety of investigations of which some are outlined in the following.

\subsubsection{Investigating Environment Impacts of ITS Solutions}

The major application is surely to measure changes in produced emissions when investigating new methods that influence traffic. In such cases, the computed emissions are used as a performance indicator besides the commonly used traffic efficiency measures, such as travel time or waiting times. Given SUMO's output capabilities, such measurements can be easily obtained.

As increasing traffic efficiency usually reduces pollutant emission, often no new insight despite obtaining numbers can be gained from such evaluations. But it is interesting to note that in some cases, the deployment of a new ITS solution may increase the amounts of produced emissions. This was shown for the GLOSA (Green Light Optimal Speed Advisory) application [20] where, when assuming long communication ranges of more than $500 \mathrm{~m}, \mathrm{a}$ vehicle may be advised to run at a low velocity (below $25 \mathrm{~km} / \mathrm{h}$ ) for a long time, yielding in 
emissions above the non-equipped situation. Therefore, it is advised to include environmental performance indicators when evaluating a new method or system.

\subsubsection{Emission-optimal Routing}

Usually, route computation is performed using travel times as weights for the edges of a road network. But what if one would use the emitted pollutants instead? Would their emission be reduced? The first investigations on this topic were performed using a real-world network [21]. To gain a deeper understanding about the dynamics of the processes, later investigations ([22]) were performed using synthetic scenarios. Neither a singular user nor a singular system optimum are assumed to be computable using currently available methods. Besides new assignment methods. But the research is as well supposed to increase the understanding about the inter-dependencies between traffic flow and pollutant emissions.

\subsubsection{Evaluation of real Traffic Management Actions}

European authorities are forced by the "Directive 2008/50/EC of the European Parliament and of the Council" to assure certain air quality. Traffic management, usually operated by local authorities, has the duty to perform corrective actions that reduce road traffic's impact, if needed. A proof-of-concept for simulating such actions that used SUMO and the HBEFA 2.1derivation is presented in [23] where three speed limit changes were investigated $-30 \mathrm{~km} / \mathrm{h}$ and $60 \mathrm{~km} / \mathrm{h}$ for urban areas and $80 \mathrm{~km} / \mathrm{h}$ for highways.

In his Master thesis ([24]), Tomàs Josep Vergés investigates the MARLIS [25] database that lists actions performed by traffic management authorities, first, to evaluate which of the actions can be simulated using only a microscopic traffic simulation. The evaluation shows that most actions target at a change in the population's mobility behavior, mainly for changing to a more environment-friendly transport mode. This can only be simulated using an according population model that was not available within his research. The resulting selection of actions to be implemented in SUMO consists of a) a reduction of the allowed velocity in inhabited areas to $30 \mathrm{~km} / \mathrm{h}, \mathrm{b}$ ) a restrictive environmental zone, and c) a permissive environmental zone. These actions are modeled, simulated using PHEMlight, and discussed within the thesis.

\subsubsection{Emissions-related COLOMBO Solutions}

The major objective of the COLOMBO project is the development of traffic management solutions that use data from vehicular communications, assuming only a small number of vehicles to be equipped with this technology. Here, not only the impacts of solutions on the environment are investigated. Instead, some parts of the work are directly concerned with the development of solutions and methods that measure or influence emission behavior. Among the targeted topics one may find a "local emissions monitoring" system that uses trajectories obtained from vehicular communications and inductive loop measures to compute the amount of emitted pollutants. A further methodology under development is the "emission optimal driver behavior" that given a vehicle and a traffic situation determines the emissionoptimal longitudinal behavior of the vehicle. COLOMBO will use the PHEMlight emission model, verifying the results against the original PHEM model.

\subsection{Summary}

Recent steps in modelling and using emissions within the open source traffic simulation SUMO were presented. As shown, the inclusion of emission models into microscopic road traffic simulations allows to gain insights into the effects of evaluated solutions on the 
environment. Even though in most cases the induction "smoother traffic -> less emissions" holds, evaluating pollutant emission behavior may offer some surprises, as named for the GLOSA example in section 1.7.1. In addition, embedding emission models into a traffic simulation allows new investigations besides measuring the impacts of ITS solutions or regulatory actions on the environment.

Three emission models that are currently implemented in SUMO were outlined. Issues regarding the first HBEFA implementation were recognized and named, and a recently implemented model that tries to solve them was described. In addition, the extension of SUMO by a commercial emission model, PHEMlight, was presented.

The presented extensions cover the work defined for the projects "COLOMBO" and "AMITRAN" well. Nonetheless, several possible extensions were already identified that may be targeted in the future. As discussed, all currently implemented models rely on the vehicle's speed, acceleration, and the slope of the road it is located at. When looking at possible future applications, the vehicle's mass comes into play. Incorporating this attribute would improve the simulation's usability for simulating fleet management and other logistics approaches. Further model improvements could be expected if the gear choice and cold-start emissions would be incorporated, but both would need new, respective models and additional input data that the user would have to supply.

But given the currently implemented models, the next steps towards a further improvements of the results should be performed on increasing the simulation's representation of single vehicles' longitudinal behaviour; it is known that nowadays car-following models neither replicate approaches to an intersections correctly nor their acceleration. As acceleration has a major influence on pollutant emissions, its correct representation should be attempted.

\section{Acknowledgements}

The authors want to thank the European Commission for co-funding the work in the context of the projects "iTETRIS", "COLOMBO", and "AMITRAN".

\section{References}

[1] International Transport Forum: REDUCING TRANSPORT GREENHOUSE GAS EMISSIONS: Trends \& Data, OECD, 2010

[2] European Parliament and the Council of the European Union, Regulation (EC) No 443/2009 setting emission performance standards for new passenger cars as part of the Community's integrated approach to reduce $\mathrm{CO} 2$ emissions from light-duty vehicles, 2009

[3] European Parliament and the Council of the European Union, Directive 2008/50/EC on ambient air quality and cleaner air for Europe, 2008

[4] Krajzewicz, D., Erdmann, J., Behrisch, M., Bieker, L.: Recent Development and Applications of SUMO - Simulation of Urban MObility, International Journal on Advances in Systems and Measurements, 5 (3\&4), pp.128-138, ISSN 1942-261x, 2012

[5] DLR and contributors: SUMO homepage, http://sumo.sourceforge.net/, 2013

[6] Krajzewicz, D., Heinrich, M., Milano, M., Bellavista, P., Stützle, T., Härri, J., Spyropoulos, T., Blokpoel, R., Hausberger, S., Fellendorf, M.: COLOMBO: Investigating 
the Potential of V2X for Traffic Management Purposes assuming low penetration Rates, In: ITS Europe 2013, Dublin

[7] COLOMBO consortium (2013): COLOMBO web pages, http://colombo-fp7.eu/, last visited on 10.04.2014

[8] Jonkers, E., Klunder, G., Mahmod, M., Benz, T.: Methodology and Framework Architecture for the Evaluation of Effects of ICT Measures on CO2 Emissions. In: Proceedings of the $20^{\text {th }}$ ITS World Congress, Tokyo, Japan, 2013

[9] AMITRAN consortium (2013): AMITRAN web pages, http://www.amitran.eu/, last visited on 10.04.2014

[10] Rondinone, M., Maneros, J., Krajzewicz, D., Bauza, R., Cataldi, P., Hrizi, F., Gozalvez, J., Kumar, V., Röckl, M., Lin, L., Lazaro, O., Leguay, J., Härri, J., Vaz, S., Lopez, Y., Sepulcre, M., Wetterwald, M., Blokpoel, R., Cartolano, F.: ITETRIS: a modular simulation platform for the large scale evaluation of cooperative ITS applications. In: Simulation Modelling Practice and Theory. Elsevier. DOI: 10.1016/j.simpat.2013.01.007. ISSN 1569-190X, 2013

[11] iTETRIS consortium: iTETRIS web site, http://www.ict-itetris.eu/, 2011, last visited on 8th of January 2014

[12] Krajzewicz, D., Blokpoel, R., Cataldi, P., Bieker, L., Ringel, J., Leguay, J., Lopez, Y.: iTETRIS Deliverable 3.2 - Traffic Modelling: ITS Algorithms, public deliverable, 2010

[13] Treiber, M., Kesting, A., Thiemann, C.: How Much does Traffic Congestion Increase Fuel Consumption and Emissions? Applying a Fuel Consumption Model to the NGSIM Trajectory Data, Presentation No. 08-2715 at the Annual Meeting of the Transportation Research Board, January 13-17, 2008, Washington, DC.

[14] Cappiello, A., Chabini, I., Nam, E.K., Lue, A., Abou Zeid, M.: A statistical model of vehicle emissions and fuel consumption, Intelligent Transportation Systems, Proceedings of the IEEE 5th International Conference on , vol., no., pp.801,809, doi: 10.1109/ITSC.2002.1041322, 2002

[15] Hausberger, S., Rexeis, M., Zallinger, M., Luz, R.(2009): Emission Factors from the Model PHEM for the HBEFA Version 3, Report Nr. I-20/2009 Haus-Em 33/08/679

[16] Technical University of Graz: pages of the Institute for Internal Combustion Engines and Thermodynamics (IVT), 2014, last visited on 10.04.2014

[17] INFRAS (2013): Handbuch für Emissionsfaktoren, http://www.hbefa.net, last visited on 06.02 .2014

[18] Hausberger, S., Krajzewicz, D.; Deliverable 4.2 - Extended Simulation Tool PHEM coupled to SUMO with User Guide, public project report, available at http://www.colombo-fp7.eu/results_deliverables.php, 2014

[19] Nota, R., Barelds, R., van Leeuwen, H.: Harmonoise WP 3 - Engineering method for road traffic and railway noise after validation and fine-tuning, Harmonoise Technical Report HAR32TR-040922-DGMR20 (Deliverable 18)

[20] Krajzewicz, D., Bieker, L., Erdmann, J.: Preparing Simulative Evaluation of the GLOSA Application. In: Proceedings CD ROM 19th ITS World Congress 2012, Paper ID: EU00630. ITS World Congress 2012, 22.-26. Okt. 2012, Wien, Austria

[21] Krajzewicz, D., Wagner, P.: Large-scale Vehicle Routing Scenarios based on Pollutant Emission. In: G. Meyer and J. Valldorf (Eds.). Advanced Microsystems for Automotive Applications 2011, AMAA 2011, Springer, 2011, pp. 237-246 
[22] Flötteröd, Y.-P., Wagner, P., Behrisch, M., Krajzewicz, D.: Simulated-based Validity Analysis of Ecological User Equilibrium. In: Winter Simulation Conference Archive, 2012 Winter Simulation Conference, 2012

[23] Krajzewicz, D., Flötteröd, Y.-P.: Simulative Untersuchung abstrakter und realer Verkehrsmanagementansätze zur Emissionsreduktion. In: Kolloquium Luftqualität an Straßen 2013, pp. 42-57. Bundesanstalt für Straßenwesen. 2013.

[24] Josep Vergés, $\mathrm{T}$ : Analysis and simulation of traffic management actions for traffic emission reduction. TU Berlin. 2013.

[25] BASt (2012): MARLIS - Datenbank mit Maßnahmen zur Reinhaltung der Luft in Bezug auf Immissionen an Straßen, Version http://www.bast.de/nn_42544/DE/Publikationen/Datenbanken/MARLIS/MARLIS.html, 06.02 .2014 